\title{
On the Performance of Lattice Reduction Schemes for MIMO Data Detection
}

\author{
Dirk Wübben \\ Department of Communications Engineering \\ University of Bremen \\ Otto-Hahn-Allee 1, D-28359 Bremen, Germany \\ Email: wuebben@ant.uni-bremen.de \\ Phone: +49 4212182407
}

\author{
Dominik Seethaler \\ Institute of Communications and Radio-Frequency \\ Engineering, Vienna University of Technology \\ Gusshausstraße 25/389, A-1040 Vienna, Austria \\ E-mail: dominik.seethaler@tuwien.ac.at \\ Phone: +4315880138958
}

\begin{abstract}
Recently the use of lattice reduction (LR) methods for data detection in multiple-input multiple-output (MIMO) systems has been proposed in order to achieve full diversity with suboptimal detection schemes. To this end, several reduction criteria and algorithms known from lattice theory have been applied. In this work new insights about the applicability of the various LR methods to linear and non-linear detection schemes are developed. In fact, the crucial performance parameters for linear and non-linear schemes are their associated postequalization SNRs. It turns out that Seysens's LR algorithm and LLL reduction perform differently with respect to these post-equalization SNRs, which explains their different error-rate behavior in context of linear as well as non-linear data detection.
\end{abstract}

Index Terms-MIMO systems, V-BLAST, spatial multiplexing, lattice-reduction, data detection, maximum likelihood.

\section{INTRODUCTION}

In the past ten years the application of multiple antennas at the transmitter and the receiver has gained considerable interest in the research community. In case of spatial multiplexing systems the source transmits parallel data streams over the antennas to increase the spectral efficiency. To estimate the transmitted data with reasonable complexity several suboptimal detection schemes have been proposed. Unfortunately, these common linear and non-linear schemes cannot fully exploit the diversity that is available in multiple-input multipleoutput (MIMO) fading channels.

In order to improve the performance of these suboptimum schemes, the application of lattice reduction (LR) as a preprocessing method has been proposed [1], [2]. In this context LR is applied to transform the system model into an equivalent one with a better conditioned channel matrix prior to low-complexity detectors using linear equalization (LE) or successive interference cancellation (SIC). This results in a significantly improved performance of the corresponding detection scheme. To this end, several reduction algorithms like the well-known LLL reduction [3] or Seysen's LR algorithm [4] have been applied for data detection in MIMO systems, e.g. [2], [5]-[9]. In fact, LLL reduction even allows LE or SIC schemes to achieve full diversity [9].

The work of D. Seethaler was supported by the STREP project MASCOT (IST-026905) within the Sixth Framework of the European Commission.
In this work new insights about the applicability of the various LR methods to LE and SIC are developed. We argue that the crucial performance parameters for LE and SIC are the corresponding post-equalization signal-to-noise ratios (SNRs) that are achieved with LR. We show that Seysen's LR algorithm and LLL reduction perform in general different with respect to these post-equalization SNRs, which explains their different error-rate behavior in combination with LE and SIC detection, respectively. For LE, Seysen's LR algorithm (or a dual version of LLL) turns out to be advantageous while for SIC detection the LLL algorithm is preferable. A general conclusion from our work is that the LR algorithm should selected taking the subsequent detector into account.

The paper is organized as follows. In Section II, the MIMO system model is introduced. The fundamentals of different LR algorithms are briefly reviewed in Section III. LR-aided detectors and their corresponding post-equalization SNRs are discussed and investigated in Section IV. Finally, the errorrate performance of various LR-aided detectors is analyzed in Section V and conclusions are provided in Section VI.

\section{SYSTEM MODEL}

We consider a spatial multiplexing MIMO system with $N_{\mathrm{T}}$ transmit and $N_{\mathrm{R}}$ receive antennas operating in a flat-fading environment. At the transmitter the data is demultiplexed into $N_{\mathrm{T}}$ parallel data streams of equal length, these substreams are mapped onto $M$-QAM symbols of the modulation alphabet $\mathcal{A}$ and transmitted over the $N_{\mathrm{T}}$ antennas simultaneously. To describe the MIMO system, one time slot of the timediscrete complex baseband model is investigated. Let ${ }^{1} \mathrm{~s}$ denote the complex-valued $N_{\mathrm{T}} \times 1$ transmit signal vector, then the corresponding $N_{\mathrm{R}} \times 1$ receive signal vector $\mathbf{x}$ is given by

$$
\mathbf{x}=\mathbf{H} \mathbf{s}+\mathbf{n},
$$

with the $N_{\mathrm{R}} \times N_{\mathrm{T}}$ channel matrix $\mathbf{H}$ and the $N_{\mathrm{R}} \times 1$ noise vector n. The noise is assumed to be complex Gaussian distributed with variance $\sigma_{n}^{2}$ and the transmit power at each antenna is normalized to one.

\footnotetext{
${ }^{1}$ The hermitian, the inverse and the pseudo-inverse are indicated by $(\cdot)^{H}$, $(\cdot)^{-1}$ and $(\cdot)^{+}$. The $i$-th column and the $i$-th row of a matrix $\mathbf{A}$ are given by $\mathbf{a}_{i}$ and $\mathbf{a}^{i)}$, respectively.
} 


\section{LATTICE REDUCTION}

In this section, we briefly review the fundamentals of various LR algorithms.

\section{A. Basic Principle of Lattice Reduction}

By interpreting the columns $\mathbf{h}_{\ell}$ of $\mathbf{H}$ as a basis and assuming an infinite $N_{\mathrm{T}}$-dimensional transmit signal space $\mathbf{s} \in \mathbb{Z}_{j}^{N_{\mathrm{T}}}$ of Gaussian integers, the set of all possible undisturbed receive signals constructs a lattice

$$
\mathcal{L}(\mathbf{H}) \triangleq \sum_{\ell=1}^{N_{\mathrm{T}}} \mathbf{h}_{\ell} \mathbb{Z}_{j} .
$$

However, the same lattice $\mathcal{L}(\tilde{\mathbf{H}})=\mathcal{L}(\mathbf{H})$ is also generated by each matrix $\tilde{\mathbf{H}}=\mathbf{H T}$, as long as the $N_{\mathrm{T}} \times N_{\mathrm{T}}$ transformation matrix $\mathbf{T}$ stems from the set of unimodular matrices $\mathrm{GL}_{N_{\mathrm{T}}}\left(\mathbb{Z}_{j}\right)$, i.e. $\mathbf{T}$ contains only Gaussian integers and the determinant is $\operatorname{det}(\mathbf{T})= \pm 1[10]$.

The general aim of LR is to transform a given basis $\mathbf{H}$ into a new basis $\tilde{\mathbf{H}}$ with vectors of shortest length or, similarly, into a basis consisting of "more orthogonal" basis vectors. Possible orthogonality measures of $\mathbf{H}$ include the condition number, the orthogonality defect, or the Seysen's measure (see Section IIID). For example, if the condition number $\kappa(\mathbf{H}) \triangleq \sigma_{\max } / \sigma_{\min }$ defined as the ratio of the largest and the smallest singular value $\sigma_{\max }$ and $\sigma_{\min }$, respectively, of $\mathbf{H}$ is equal to one, then $\mathbf{H}$ is orthogonal. It is well-known, that $\kappa(\mathbf{H})$ describes the impact of the channel realization on the noise enhancement in case of LE (see e.g. [11]). A new basis may achieve a smaller $\kappa(\mathbf{H})$ and, consequently, better performance results in the case of LE, as the impact of noise enhancement is reduced.

\section{B. LLL-Reduction}

The original definition of LLL reduction by Lenstra, Lenstra and Lovász was restricted to real-valued matrices [3] and extended with respect to complex matrices recently [7]. Within this paper we focus on its complex-valued version since it requires less complexity and achieves comparable performance results. The LLL algorithm is based on the $\mathrm{QR}$ decomposition (QRD) $\mathbf{H}=\mathbf{Q R}$, where matrix $\mathbf{Q} \in \mathbb{C}^{N_{\mathrm{R}} \times N_{\mathrm{T}}}$ has orthogonal columns of unit length and $\mathbf{R}=\left[r_{k, \ell}\right]_{1 \leq k, \ell \leq N_{\mathbb{T}}}$ is an upper triangular matrix. With respect to the $\tilde{Q} R \overline{\mathbf{H}}=\tilde{\mathbf{Q}} \tilde{\mathbf{R}}$ of the reduced basis, the basis vector $\tilde{\mathbf{h}}_{\ell}$ is almost orthogonal to $\operatorname{span}\left\{\tilde{\mathbf{h}}_{1}, \ldots, \tilde{\mathbf{h}}_{\ell-1}\right\}$, if the elements $\left|\tilde{r}_{1, \ell}\right|, \ldots,\left|\tilde{r}_{\ell-1, \ell}\right|$ of $\tilde{\mathbf{R}}$ are close to zero. Using this observation the LLL algorithm can be stated as follows.

Definition (LLL-Reduced): A basis $\tilde{\mathbf{H}}$ with QRD $\tilde{\mathbf{H}}=$ $\tilde{\mathbf{Q}} \tilde{\mathbf{R}}$ is called LLL-reduced with parameter $1 / 4<\delta \leq 1$, if

$$
\begin{aligned}
\left|\tilde{r}_{k, \ell}\right| & \leq \frac{1}{2}\left|\tilde{r}_{k, k}\right| & \text { for } 1 & \leq k<\ell \leq N_{\mathrm{T}} \\
\delta\left|\tilde{r}_{k-1, k-1}\right|^{2} & \leq\left|\tilde{r}_{k, k}\right|^{2}+\left|\tilde{r}_{k-1, k}\right|^{2} & \text { for } k & =2, \ldots, N_{\mathrm{T}}
\end{aligned}
$$

holds.

Starting with $\mathbf{H}=\mathbf{Q R}$ the iterative LLL algorithm yields a reduced basis $\tilde{\mathbf{H}}=\tilde{\mathbf{Q}} \tilde{\mathbf{R}}$ and a corresponding transformation matrix T. To this end, the algorithm successively performs so-called size-reductions to meet (3a) and exchanges two neighboring basis vectors if (3b) is not fulfilled [3], [5].

The beneficial impact of applying the Sorted QR Decomposition (SQRD) [12] to the computational complexity of the LLL algorithm was presented in [5], [6]. Furthermore it was shown, that the consideration of the MMSE criterion by reducing the extended channel matrix

$$
\underline{\mathbf{H}} \triangleq\left[\begin{array}{c}
\mathbf{H} \\
\sigma_{n} \mathbf{I}_{N_{\mathrm{T}}}
\end{array}\right]
$$

leads simultaneously to a strong performance improvement and further reduction of computational complexity [5].

\section{Dual LLL-Reduction}

Instead of performing LLL reduction with respect to the basis $\mathbf{H}$, a reduction of the dual basis

$$
\mathbf{H}^{\star} \triangleq\left(\mathbf{H}^{+}\right)^{H}=\mathbf{H}\left(\mathbf{H}^{H} \mathbf{H}\right)^{-1}
$$

was proposed in [9] in order to improve the performance of LR-aided LE. By applying LLL with respect to the dual basis the QR decomposition $\tilde{\mathbf{H}}^{\star}=\tilde{\mathbf{Q}}^{\star} \tilde{\mathbf{R}}^{\star}$ is achieved. In the sequel, this reduction will be abbreviated by DLLL and the corresponding $\ell$-th dual basis vector is denoted with $\mathbf{h}_{\ell}^{\star}$. For MMSE the extended dual matrix $\underline{\mathbf{H}}^{\star}=\left(\underline{\mathbf{H}}^{+}\right)^{H}$ is reduced.

\section{Seysen's LR Algorithm}

The basic principle of Seyen's LR algorithm [4] lies in the simultaneous reduction of the basis $\mathbf{H}$ and the dual basis $\mathbf{H}^{\star}$. To this end, Seysen introduced the following orthogonality criteria.

Definition ( $S$-Reduced): A basis $\mathbf{H}$ is called $S$-reduced if $S(\mathbf{H}) \leq S(\mathbf{H T})$ holds for all $\mathbf{T} \in \mathrm{GL}_{N_{\mathrm{T}}}\left(\mathbb{Z}_{j}\right)$ with Seysen's measure $S(\mathbf{H})$ defined by

$$
S(\mathbf{H}) \triangleq \sum_{\ell=1}^{N_{\mathrm{T}}}\left\|\mathbf{h}_{\ell}\right\|^{2}\left\|\mathbf{h}_{\ell}^{\star}\right\|^{2} .
$$

Thus, for an $S$-reduced basis, no unimodular transformation matrix $\mathbf{T}$ leads to a further reduction of Seysen's measure $S(\mathbf{H})$. We have $S(\mathbf{H}) \geq N_{\mathrm{T}}$ and its minimum $N_{\mathrm{T}}$ is achieved if and only if the basis is orthogonal. Common, suboptimum algorithms perform a successive sequence of elementary column operations with respect to just two basis vectors, leading to the so-called $S_{2}$-reduction (referred to as Seysen's LR algorithm or briefly SEY in the sequel) [4]. The application of SEY for symbol detection and an efficient implementation of the reduction algorithm have recently been proposed in [8]. Therein it is demonstrated that SEY can find more orthogonal bases (with respect to various orthogonality measures) than the LLL algorithm by requiring less basis updates.

\section{LR-Aided Detection SCHEMES AND THEIR POST-EQUALIZATION SNRS}

In the sequel we assume that the reduced basis and transformation matrix have been calculated either by SEY, LLL, or DLLL. The obtained result is then used for LE or SIC 
detection. In the following, we describe these LR-aided ZeroForcing (ZF) detectors and discuss the impact of the corresponding post-equalization SNRs on the performance of theses schemes. However, the argumentation can easily extended for the SINRs of MMSE detection by consideration of the extended channel matrix (4).

\section{A. LR-Aided Data Detection}

By using the reduced channel matrix $\tilde{\mathbf{H}}=\mathbf{H T}$ and introducing $\mathbf{z}=\mathbf{T}^{-1} \mathbf{s}$, system model (1) can be rewritten as [6]

$$
\mathbf{x}=\mathbf{H} \mathbf{s}+\mathbf{n}=\mathbf{H T T}^{-1} \mathbf{s}+\mathbf{n}=\tilde{\mathbf{H}} \mathbf{z}+\mathbf{n} .
$$

The idea behind LR-aided data detection is to consider this equivalent system model with reduced channel matrix $\tilde{\mathbf{H}}$ and perform the detection with respect to $\mathbf{z}$ instead of $\mathbf{s}$. Indeed, if $\mathbf{s} \in \mathbb{Z}_{j}^{N_{\mathrm{T}}}$, we also have $\mathbf{z} \in \mathbb{Z}_{j}^{N_{\mathrm{T}}}$ since $\mathbf{T}$ is unimodular. Thus, one can first calculate a detection result for $\mathbf{z}$, denoted with $\hat{\mathbf{z}}$, and then calculate $\hat{\mathbf{s}}=\mathbf{T} \hat{\mathbf{z}}$, which represents the final detection result for the original problem. A corresponding block diagram is shown in Fig. 1. The case of finite constellations is discussed in detail in [5].

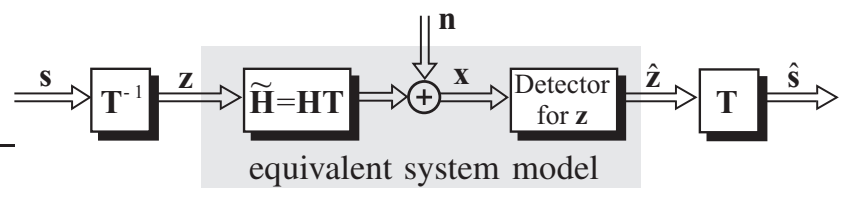

Fig. 1. Block diagram of LR-aided data detection.

\section{B. LR-Aided LE}

For LR-aided Zero-Forcing (ZF) LE, first $\tilde{\mathbf{z}}=\mathbf{G x}$ with filter matrix

$$
\mathbf{G}=\tilde{\mathbf{H}}^{+}=\left(\tilde{\mathbf{H}}^{H} \tilde{\mathbf{H}}\right)^{-1} \tilde{\mathbf{H}}^{H}
$$

is calculated, where $\mathbf{G}$ is given by the pseudo-inverse of $\tilde{\mathbf{H}}$. The hard decision $\hat{\mathbf{z}}$ is then obtained through (usually unconstrained) componentwise quantization of $\tilde{\mathbf{z}}$, i.e. $\hat{\mathbf{z}}=$ $\mathcal{Q}\{\tilde{\mathbf{z}}\}$. The multiplication with $\tilde{\mathbf{H}}^{+}$usually causes less noise amplification than the multiplication with $\mathbf{H}^{+}$due to the more orthogonal columns of $\tilde{\mathbf{H}}$. Therefore, a hard decision based on $\tilde{\mathbf{z}}$ is in general more reliable than that based on $\tilde{\mathbf{s}}=\mathbf{H}^{+} \mathbf{x}$ (corresponding to conventional ZF-LE). As mentioned before, the extension with respect to the MMSE criterion is directly achieved by LR of the extended channel matrix $\underline{\mathbf{H}}$ introduced in (4). As demonstrated in [5], [6] this leads to significant performance improvements and reduction in complexity.

1) Performance Analysis: To analyze the performance of LR-aided LE we consider the corresponding post-equalization SNR of layer $\ell, \ell=1, \ldots, N_{\mathrm{T}}$. After ZF equalization, we have $\tilde{\mathbf{z}}=\mathbf{z}+\mathbf{G n}$, which is used to form the hard decision $\hat{\mathbf{z}}$ in the transformed domain. Evidently, the effective noise of layer $\ell$ is complex Gaussian distributed with variance $\sigma_{n}^{2}\left\|\mathbf{g}^{(\ell)}\right\|^{2}$, where $\mathrm{g}^{(\ell)}$ denotes the $\ell$-th row of the filter matrix $\mathbf{G}$. By recalling the definition of the dual basis (5), we thus have

$$
\mathrm{SNR}_{\ell}^{\mathrm{LE}} \triangleq \frac{1}{\sigma_{n}^{2}\left\|\mathbf{g}^{(\ell)}\right\|^{2}}=\frac{1}{\sigma_{n}^{2}\left\|\mathbf{h}_{\ell}^{\star}\right\|^{2}} .
$$

We see that the performance of LE is determined by the Euclidian lengths of the dual basis vectors (see also [9]). Furthermore, the overall error probability will be dominated by the layer $\ell$ with the minimum $\mathrm{SNR}_{\ell}^{\mathrm{LE}}$, i.e. the worst layer (or subchannel). Thus, for LE it is reasonable to determine a reduced basis where the corresponding longest dual vector is as short as possible or the minimum $\mathrm{SNR}_{\ell}^{\mathrm{LE}}$ is maximized. Note that this does not necessarily correspond to the standard target of LR methods (e.g. to find the shortest basis vector).

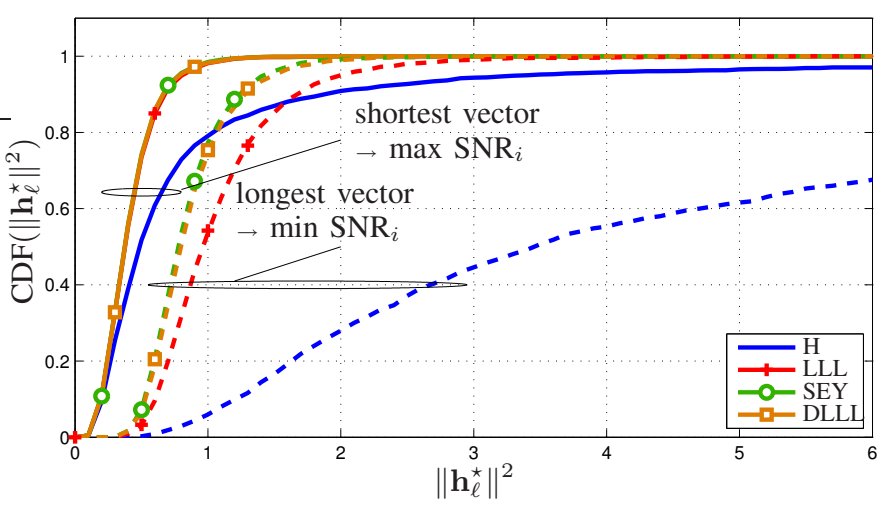

Fig. 2. CDF of squared length of shortest (-) and longest (--) dual basis vector for a system with $N_{\mathrm{T}}=N_{\mathrm{R}}=6$ antennas.

Fig. 2 shows the cumulative distribution functions (CDFs) of the longest and the shortest filter (i.e. dual basis) vectors of the original and the reduced case for a MIMO system with $N_{\mathrm{R}}=N_{\mathrm{R}}=6$ antennas. All LR schemes lead to an almost equivalent reduction of the shortest vector. However, with respect to the performance dominating longest dual basis vector, SEY and DLLL outperform LLL. For LE, these LR schemes indeed show better performance results (see Section V).

2) Computational Effort: With respect to computational complexity it is worth to note that the filter matrix $\mathbf{G}$ is already calculated for DLLL and SEY, as both methods calculate the reduced dual basis $\tilde{\mathbf{H}}^{\star}=\mathbf{G}^{H}$. As the LLL is executed with respect to the $\mathrm{QR}$ decomposition of $\tilde{\mathbf{H}}$, the required pseudoinverse can be determined by $\mathbf{G}=\tilde{\mathbf{H}}^{+}=\tilde{\mathbf{R}}^{-1} \tilde{\mathbf{Q}}^{H}$.

\section{LR-Aided SIC}

The performance of LE can be improved by applying SIC (or decision-feedback) techniques based on the QR decomposition $\tilde{\mathbf{H}}=\tilde{\mathbf{Q}} \tilde{\mathbf{R}}$. We obtain

$$
\tilde{\mathbf{z}}=\tilde{\mathbf{Q}}^{H} \mathbf{x}=\tilde{\mathbf{R}} \mathbf{z}+\tilde{\mathbf{n}},
$$

where $\tilde{\mathbf{n}}=\tilde{\mathbf{Q}}^{H} \mathbf{n}$. Due to the upper triangular form of $\tilde{\mathbf{R}}$ the vector $\tilde{\mathbf{z}}$ is partly free of interference and can successively be detected layer by layer in the sequence $\ell=N_{\mathrm{T}}, N_{\mathrm{T}}-1, \ldots, 1$. In order to estimate $z_{\ell}$, previous decisions for $\hat{z}_{\ell+1}, \ldots, \hat{z}_{N_{\mathrm{T}}}$ weighted by the corresponding coefficients of $\tilde{\mathbf{R}}$ are subtracted from $\tilde{z}_{\ell}$

$$
\hat{z}_{\ell}=\mathcal{Q}\left\{\frac{1}{\tilde{r}_{\ell, \ell}}\left(\tilde{z}_{\ell}-\sum_{k=\ell+1}^{N_{\mathrm{T}}} \tilde{r}_{\ell, k} \cdot \hat{s}_{k}\right)\right\} .
$$


1) Performance Analysis: Assuming correct decisions in previous layers, the post-equalization SNR at the $\ell$-th layer (respectively, $\ell$-th detection step) is proportional to the squared value of the $\ell$-th diagonal element $\tilde{r}_{\ell, \ell}$ of the upper triangular matrix $\tilde{\mathbf{R}}$. To be more precise, we have

$$
\mathrm{SNR}_{\ell}^{\mathrm{SIC}}=\sigma_{n}^{-2}\left|\tilde{r}_{\ell, \ell}\right|^{2} .
$$

Note that $\mathrm{SNR}_{N_{\mathrm{T}}}^{\mathrm{SIC}}=\mathrm{SNR}_{N_{\mathrm{T}}}^{\mathrm{LE}}$ and $\mathrm{SNR}_{\ell}^{\mathrm{SIC}} \neq \mathrm{SNR}_{\ell}^{\mathrm{LE}}$ for $\ell<N_{\mathrm{T}}$ in general for SIC without optimized detection ordering. It is well known that the first layer in the detection and interference cancellation procedure $\left(\ell=N_{\mathrm{T}}\right)$ dominates the overall performance. This is due to the fact that the first detection step degrades the subsequent decisions through error propagation (cf. (11)). Consequently, it is reasonable to find a reduced basis such that $\mathrm{SNR}_{N_{\mathrm{T}}}^{\mathrm{SIC}}$, or equivalently, $\left|\tilde{r}_{N_{\mathrm{T}}, N_{\mathrm{T}}}\right|^{2}$, is as large as possible. Evidently, we arrived at an LR performance criterion for SIC that is in general different from that for LE, where the minimum $\mathrm{SNR}_{\ell}^{\mathrm{LE}}$ should be as large as possible (see previous Section). Furthermore, layer ordering approaches [12] have a similar target, however, by just allowing column permutations. In fact, permuting columns is the simplest form of changing the basis of a given lattice and consequently can be regarded as a part of LR (or LR for SIC can be seen as "extended layer ordering"). Of course, layer ordering does not improve the performance of LE.

a) CDF of $\left|r_{6,6}\right|^{2}$ w/o sorting
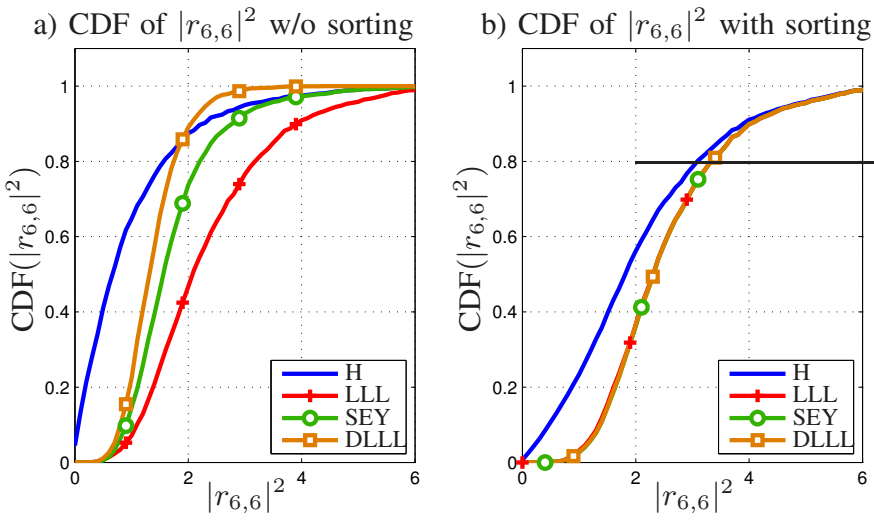

Fig. 3. CDF of $\left|r_{6,6}\right|^{2}$ a) without and b) with optimized detection ordering for a system with $N_{\mathrm{T}}=N_{\mathrm{R}}=6$ antennas.

For a MIMO systems with $N_{\mathrm{T}}=N_{\mathrm{R}}=6$ antennas part a) of Fig. 3 shows the CDFs of $\left|r_{6,6}\right|^{2}$ for the different LR schemes. Obviously, the LLL achieves the best statistic for $\left|r_{6,6}\right|^{2}$ and would outperform SEY and DLLL reduction with respect to error-rate due to the improved first detection step. As the LLL successively reduces the elements $r_{\ell, \ell}$, it directly leads to larger SNRs in the first detection steps and thereby to a meaningful detection sequence. In contrast, SEY leads to a balanced reduction of all basis vectors without any ordering. Part b) of Fig. 3 shows the CDFs of $\left|r_{6,6}\right|^{2}$ for different LR algorithms with optimum layer ordering using the post-sorting algorithm of [12]. It can be seen that with post-sorting all LR schemes lead to approximately the same statistic for $\left|r_{6,6}\right|^{2}$. Consequently, similar performance results can be expected for the corresponding LR-aided SIC schemes (see Section V).
2) Computational Effort: In case LLL is used for reduction, the required QRD is implicitly available. In contrast, for SEY the corresponding QRD of $\tilde{\mathbf{H}}$ has to be calculated afterwards and leads thereby to an additional computational effort. In case of DLLL, the QRD of the dual basis $\tilde{\mathbf{H}}^{\star}=\tilde{\mathbf{Q}}^{\star} \tilde{\mathbf{R}}^{\star}$ is available. For SIC detection, one can either calculate the QRD of $\left(\tilde{\mathbf{H}}^{\star}\right)^{+}$ or implement SIC with respect to a QL decomposition, where the matrix with orthogonal columns is given by $\left(\left(\tilde{\mathbf{Q}}^{\star}\right)^{+}\right)^{H}$ and $\left(\left(\tilde{\mathbf{R}}^{\star}\right)^{+}\right)^{H}$ describes the corresponding lower triangular matrix. Obviously, both strategies result in a considerable additional complexity. To summarize, for SIC detection based on QRD, it is favorable from an implementation point of view to use the LLL algorithm for LR.

\section{Performance Results of LR-Aided Detectors}

In the sequel we investigate the bit error-rate (BER) performance of various LR-aided detectors for a MIMO system with $N_{\mathrm{T}}=N_{\mathrm{R}}=6$ antennas employing 4-QAM modulation. The SNR is given by $E_{b} / N_{0}=N_{\mathrm{R}} /\left(\log _{2}(M) \sigma_{n}^{2}\right)$ with $E_{b}$ denoting the average energy per information bit arriving at the receiver.

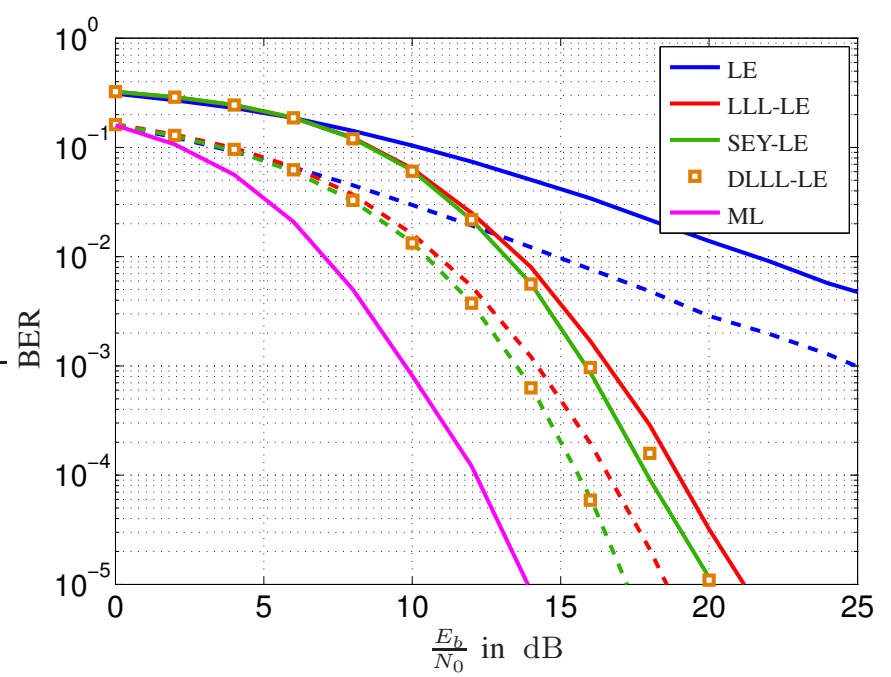

Fig. 4. BER versus $E_{b} / N_{0}$ performance of common and LR-aided LE detectors for a MIMO system with $N_{\mathrm{T}}=N_{\mathrm{R}}=6$ antennas employing 4-QAM symbols: ZF (-) and MMSE (--).

Fig. 4 shows the BER versus $E_{b} / N_{0}$ performance of common and LR-aided LEs. It can be seen that the LRaided LEs achieve full diversity (at least for LLL and DLLL this is proven in [9]) thereby leading to strong performance improvements compared to the common LE. Furthermore, the MMSE criterion results in additional performance gains. When comparing the LR schemes for LE, we can observe that SEY and DLLL clearly outperform LLL. This performance difference can be explained by the observations made in Section IV-B. On average, both SEY and DLLL achieve a stronger reduction of the longest dual basis vector leading to an improved worst-case post-equalization SNR. We thus observe that it is more important to reduce the lengths of the dual basis vectors (in particular, the length of the longest dual vector) instead of shortening the original basis (or finding its shortest basis vector) in the case of LE. 


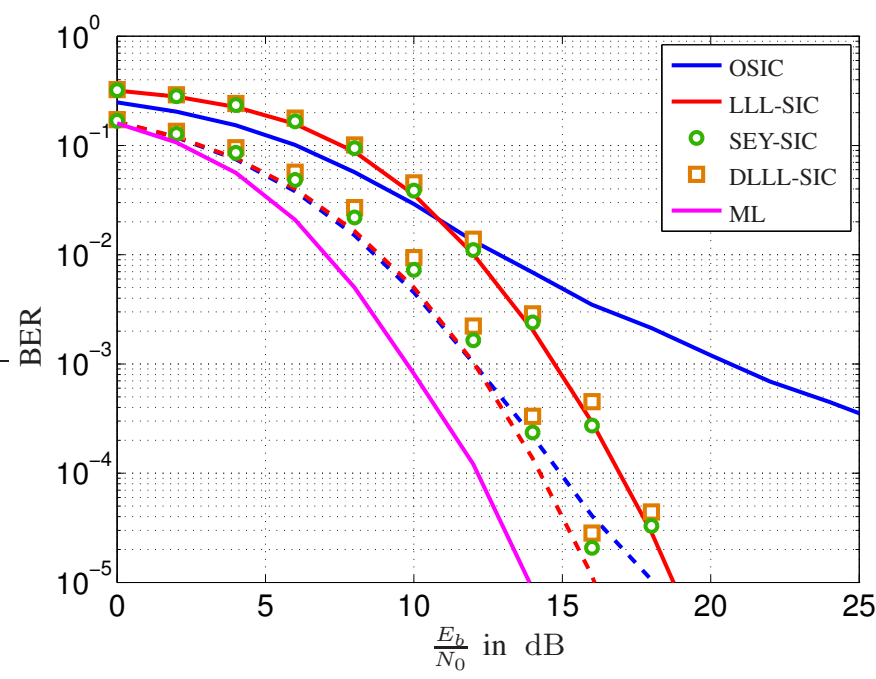

Fig. 5. BER versus $E_{b} / N_{0}$ performance of common and LR-aided SIC detectors for a MIMO system with $N_{\mathrm{T}}=N_{\mathrm{R}}=6$ antennas employing 4-QAM symbols: ZF (-) and MMSE (- -).

The BER versus $E_{b} / N_{0}$ performance of ordered SIC (OSIC) and LR-aided conventional SIC detection (without applying post-sorting after lattice reduction) are shown in Fig. 5. Obviously, LLL-aided SIC slightly outperforms the other two LR-aided SIC methods (SEY-SIC and DLLL-SIC). However, as demonstrated in Fig. 6 these differences disappear if SIC detection with optimization of the detection order is applied (cf. Section IV-C).

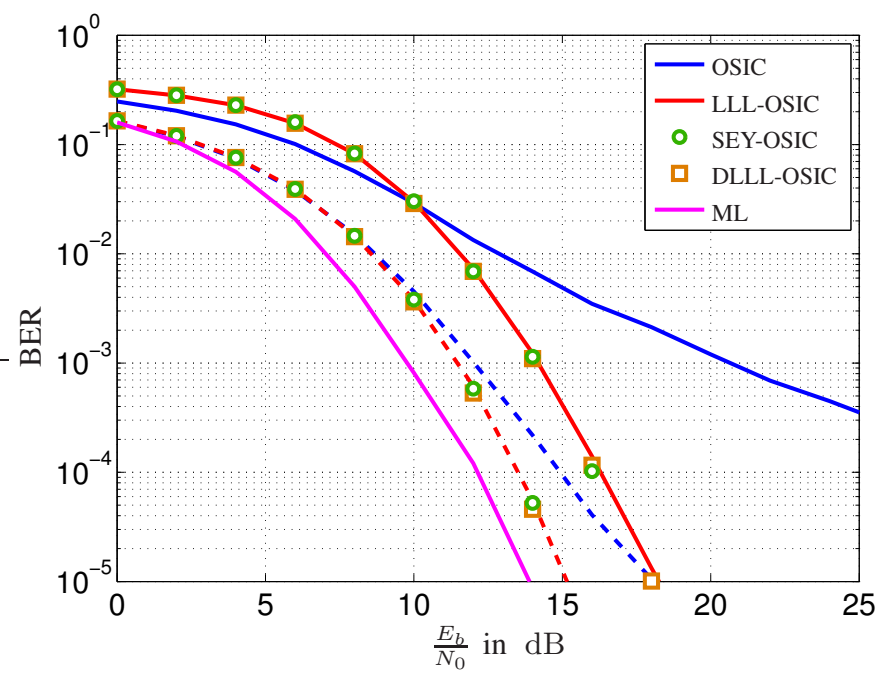

Fig. 6. BER versus $E_{b} / N_{0}$ performance of common and LR-aided optimally ordered SIC (OSIC) detectors for a MIMO system with $N_{\mathrm{T}}=N_{\mathrm{R}}=6$ antennas employing 4-QAM symbols: ZF (-) and MMSE (--).

Thus, from the performance point of view the different LRschemes behave almost similar when post-sorting is applied. However, as discussed in Section IV-C the required QRD with a good detection sequence is directly obtained by LLL reduction. Consequently, for SIC detection the application of LLL is favorable with respect to complexity.

\section{Summary AND CONCLUSIONS}

In this paper, we investigated lattice reduction (LR) methods for data detection in MIMO wireless systems. In particular, we focused on three LR methods (namely, the LLL algorithm, the LLL algorithm applied to the dual basis, and Seysen's algorithm) and two detection approaches (namely, linear equalization (LE) and non-linear successive interference cancellation (SIC)). We argued that the LR performance requirements for LE and SIC are in general different. For LE the longest basis vector of the dual basis should be as short as possible and for SIC the post-equalization SNR of the first layer in the detection progress should be as large as possible. We demonstrated that (dual) LLL and Seysen's algorithm perform differently with respect to these requirements, which provides an explanation for their different error performance in combination with LE and SIC. In case of LE, dual LLL and Seysen's algorithm give the best performance. For SIC detection, however, all investigated LR schemes can achieve comparable performance results with a slight advantage of LLL. In general, an LR algorithm should selected (or designed) taking the the subsequent detection strategy into account.

\section{REFERENCES}

[1] H. Yao and G. W. Wornell, "Lattice-Reduction-Aided Detectors for MIMO Communication Systems," in IEEE Proc. Global Communications Conference (GLOBECOM), Taipei, Taiwan, Nov. 2002.

[2] C. Windpassinger and R. F. H. Fischer, "Low-Complexity NearMaximum-Likelihood Detection and Precoding for MIMO Systems using Lattice Reduction," in IEEE Proc. Information Theory Workshop (ITW), Paris, France, Mar. 2003.

[3] A. K. Lenstra, H. W. Lenstra, and L. Lovász, "Factoring Polynomials with Rational Coefficients," Mathematische Annalen, vol. 261, pp. 515534, 1982.

[4] M. Seysen, "Simultaneous Reduction of a Lattice Basis and its Reciprocal Basis," Combinatorica, vol. 13, pp. 363-376, 1993.

[5] D. Wübben, R. Böhnke, V. Kühn, and K.-D. Kammeyer, "MMSE-Based Lattice Reduction for Near-ML Detection of MIMO Systems," in ITG Proc. Workshop on Smart Antennas (WSA), Munich, Germany, Mar. 2004.

[6] — - "Near-Maximum-Likelihood Detection of MIMO Systems using MMSE-Based Lattice Reduction," in IEEE Proc. International Conference on Communications (ICC), Paris, France, June 2004, pp. 798-802.

[7] Y. H. Gan and H. W. Mow, "Complex Lattice Reduction Algorithms for Low-Complexity MIMO Detection," in IEEE Proc. Global Communications Conference (GLOBECOM), St. Louis, MI, USA, Nov. 2005.

[8] D. Seethaler, G. Matz, and F. Hlawatsch, "Low-Complexity MIMO Detection using Seysen's Lattice Reduction Algorithm," in IEEE Proc. International Conference on Acoustics, Speech, and Signal Processing (ICASSP), Honolulu, HI, USA, Apr. 2007.

[9] M. Taherzadeh, A. Mobasher, and A. K. Khandani, "LLL Reduction Achieves the Receive Diversity in MIMO Decoding," IEEE Trans. on Information Theory, to appear.

[10] J. von zur Gathen and J. Gerhard, Modern Computer Algebra, 2nd ed. Cambridge, UK: Cambridge University Press, 2003.

[11] H. Artés, D. Seethaler, and F. Hlawatsch, "Efficient Detection Algorithms for MIMO Channels: A Geometrical Approach to Approximate ML Detection," IEEE Trans. Signal Processing, vol. 51, no. 11, pp. 2808-2820, Nov. 2003.

[12] D. Wübben, R. Böhnke, V. Kühn, and K.-D. Kammeyer, "MMSE Extension of V-BLAST based on Sorted QR Decomposition," in IEEE Proc. Vehicular Technology Conference (VTC), Orlando, Florida, USA, Oct. 2003. 